\title{
Lensing of fast radio bursts by binaries to probe compact dark matter
}

\author{
Y. K. Wang ${ }^{1}$ and F. Y. Wang ${ }^{1,2}$ \\ ${ }^{1}$ School of Astronomy and Space Science, Nanjing University, Nanjing 210093, PR China \\ e-mail: fayinwang@nju.edu.cn \\ 2 Key Laboratory of Modern Astronomy and Astrophysics, Nanjing University, Ministry of Education, Nanjing 210093, PR China
}

Received 12 May 2017 / Accepted 20 January 2018

\begin{abstract}
The possibility that a fraction of dark matter is comprised of massive compact halo objects (MACHOs) remains unclear, especially in the 20-100 $M_{\odot}$ window. MACHOs could make up binaries, whose mergers may be detected by LIGO as gravitational wave events. On the other hand, the cosmological origin of fast radio burst (FRBs) has been confirmed. We investigate the possibility of detecting FRBs gravitational lensed by MACHO binaries to constrain their properties. Since lensing events could generate more than one image, lensing by binaries could cause multiple-peak FRBs. The angular separation between these images is roughly $10^{-3}$ mas, which is too small to be resolved. The typical time interval between different images is roughly 1 millisecond (ms). The flux ratio between different images is from approximately 10 to $10^{3}$. With the expected detection rate of $10^{4} \mathrm{FRBs}$ per year by the upcoming experiments, we could expect five multi-peak FRBs observed per year with a time interval larger than $1 \mathrm{~ms}$ and flux ratio less than $10^{3}$ if the fraction of dark matter in MACHOs is $f \sim 0.01$. A null search of multiple-peak FRBs for time intervals larger than $1 \mathrm{~ms}$ and flux ratio less than $10^{3}$ with $10^{4}$ FRBs would constrain the fraction $f$ of dark matter in MACHOs to $f<0.001$.
\end{abstract}

Key words. gravitational lensing: strong - dark matter

\section{Introduction}

The nature of dark matter is an important question in cosmology. Among many candidates, the weakly interacting massive particle (WIMP) is a popular hypothesis but has not been proved. Alternatively, it has been proposed that massive compact halo objects (MACHOs) could make up the dark matter in our Universe (Carr \& Hawking 1974; Carr 1975, 1976; Mészáros 1975; García-Bellido et al. 1996; Khlopov 2010; Frampton et al. 2010; Belotsky et al. 2014). Many experiments have put constraints on the fraction of dark matter that can reside in MACHOs with a given mass. Low-mass MACHOs $\left(<20 M_{\odot}\right)$ are ruled out by microlensing surveys since they would cause luminosity variability in stars (Alcock et al. 2001; Wyrzykowski et al. 2011; Tisserand et al. 2007). High-mass MACHOs (>100 $\left.M_{\odot}\right)$ are excluded because they would disrupt wide binaries (Yoo et al. 2004; Quinn et al. 2009; Monroy-Rodríguez \& Allen 2014). The remaining 20-100 $M_{\odot}$ window is argued to be constrained by cosmic microwave background (CMB), because the process of gas accretion onto primordial black holes in the early Universe could modify the cosmic recombination history, which would affect the spectrum and anisotropies of CMB (Ricotti 2007; Ricotti et al. 2008). However, a significant uncertainty is associated with this constraint (Ali-Haïmoud \& Kamionkowski 2017) leaving the possibility that part of the dark matter could still be made of MACHOs (Pooley et al. 2009; Mediavilla et al. 2009).

Recently, the advanced Laser Interferometer Gravitationalwave Observatory (LIGO) detectors recorded the first gravitational wave (GW) event GW150914 (Abbott et al. 2016a), and the second GW 151226 (Abbott et al. 2016b). Both events are produced by the mergers of black hole binaries, each with a mass of about $30 M_{\odot}$. However, in the traditional stellar evolution scenario, binaries of black holes with masses of about $30 M_{\odot}$ each are very difficult to form. Some models have been proposed for the progenitors of binary black hole systems (Hosokawa et al. 2016; Belczynski et al. 2016; Rodriguez et al. 2016a,b; Woosley 2016; Chatterjee et al. 2017; Mink \& Mandel 2016; Hartwig et al. 2016; Inayoshi et al. 2016; Arvanitaki et al. 2017). More interestingly, it has also been proposed that GW events could possibly be the mergers of primordial black holes (PBHs; Bird et al. 2016; Sasaki et al. 2016), which could be treated as a part of MACHOs.

Several ideas have been proposed to test the possibility that the mergers of PBHs could be the progenitors of gravitational wave events. It was suggested that the detectable residual eccentricity when the two PBHs merge could help us test this possibility (Cholis et al. 2016). If GW events are caused by the mergers of PBHs, the GW events should have a low correlation with luminous galaxies. The measurements of the cross-correlation of the GW events with overlapping galaxy catalogs could help us test the model (Raccanelli et al. 2016). The possibility of detecting stochastic GW background from mergers of PBHs has been investigated (Mandic et al. 2016). Strong gravitational lensing of extragalactic fast radio bursts (FRBs) by PBHs is suggested to test the model (Muñoz et al. 2016).

FRBs are bright bursts of radio emission with a duration of a few milliseconds. The dispersion measures (DMs) of FRBs are much larger than the line of sight DM contribution expected from the electron distribution of our Galaxy. Recently, the precise localization of the repeating FRB 121102 has revealed that it resides in a dwarf galaxy at a redshift of $z=0.19$ (Chatterjee et al. 2017; Tendulkar et al. 2016), confirming its cosmological origin. FRBs are proposed to measuring the cosmic proper 
distance Yu \& Wang (2017). Many theoretical models for FRBs are proposed, such as collapses of supra-massive neutron stars (Falcke \& Rezzolla 2014; Zhang 2014), charged black hole binary mergers (Zhang 2016), collisions between asteroids and a high magnetized pulsar (Dai et al. 2016), superconducting cosmic strings (Yu et al. 2014) and double neutron star mergers (Totani 2013; Wang et al. 2016).

In this paper, we present the gravitational lensing properties of fast radio bursts (FRBs) by $\mathrm{PBH}$ binaries and probe MACHOs. FRBs are ideal sources to detect lensing events since they are strong and short and occur at a rate about a few thousands per day for the whole sky (Lorimer et al. 2007; Thornton et al. 2013). It has been proposed that strong lensing of FRBs by isolated PBHs could be used to probe MACHOs $\left(>20 M_{\odot}\right)$ (Muñoz et al. 2016). Considering that the three GW events are produced by mergers of black hole binaries, we study the strong lensing of FRBs by PBH binaries. In this case, we could detect multiple-peak FRBs, unlike the repeating FRBs. Since FRBs are strong, short bursts, their multi-peak structure might be observable. In addition, upcoming or ongoing surveys like APERTIF (Verheijen et al. 2008), UTMOST (Caleb et al. 2016), HIRAX (Newburgh et al. 2016), and CHIME (Bandura et al. 2014) expect to detect a large number of FRBs, providing a chance to detect multiple-peak FRBs. We adopted the distribution of the separations of PBH binaries in Nakamura et al. (1997) and Sasaki et al. (2016), which assumes that PBHs' velocities relative to the primordial gas have been redshifted to negligible speeds when the gravitational attractions become important. Without perturbation, two nearby black holes would merge directly. However, the tidal force from other PBHs could provide angular momentum for the two black holes to form a binary system.

This paper is organized as follows. In Sect. 2, we present the theories on strong lensing by binaries and give our calculation on the optical depth for lensing by $\mathrm{PBH}$ binaries. In Sect. 3, we introduce our simulation of the lensing events of FRBs by PBH binaries. Discussions and conclusion are given in Sect. 4.

\section{Optical depth for lensing by PBH binaries}

The theory of lensing by two-point-mass lenses is discussed in detail by Schneider \& Weiss (1986). In the case of FRBs lensed by PBH binaries, we treat two PBHs as two point lenses. $M=$ $M_{1}+M_{2}$ is defined as the total mass of the lenses, where $M_{1}$ and $M_{2}$ are the masses of two separate lenses. Throughout the paper, we consider the case $M_{1}=M_{2}=30 M_{\odot}$ as an example. Then the Einstein radius is defined as

$\beta_{\mathrm{E}}=\sqrt{\frac{4 G M}{c^{2}} \frac{D_{\mathrm{d}} D_{\mathrm{ds}}}{D_{\mathrm{s}}}}$,

where $D_{\mathrm{d}}$ is the angular diameter distance from the lens to the observer, $D_{\mathrm{s}}$ is the angular diameter distance from the source to the observer, and $D_{\mathrm{ds}}$ is the angular diameter distance between lens and source. We define the following dimensionless quantities

$r=\xi / \beta_{\mathrm{E}}$

$x=\left(\frac{D_{\mathrm{d}}}{D_{\mathrm{s}}}\right) \eta / \beta_{\mathrm{E}}$,

$\mu_{1}=M_{1} / M$,

$\mu_{2}=M_{2} / M$, where $\eta$ is the position of the source in the source plane, and $\xi$ is the position where light rays hit the lens plane.

Given the position of the source, there are between three and five image positions. The positions of images in the lens plane are given by the lens equation (Schneider \& Weiss 1986),

$x=r-\alpha(r)$,

where

$\alpha(r)=\mu_{1} \frac{r-r_{0}}{\left|r-r_{0}\right|^{2}}+\mu_{2} \frac{r+r_{0}}{\left|r+r_{0}\right|^{2}}$.

Lenses are located at the points $\beta_{\mathrm{E}} r_{0}$ and $-\beta_{\mathrm{E}} r_{0} . r_{0}=(X, 0)$, where $X \geq 0$ is the position of the lens on the lens plane.

With the positions of lenses, source and images, we can calculate the amplification factor $I_{0}$ for every image, that is, the ratio of the flux density of one of the images caused by light deflection and the image when the lenses were absent

$I_{0}=|\operatorname{det} A|^{-1}$,

where $A=\partial x / \partial r$, and

$\operatorname{det} A=1-\left(\frac{\mu_{1}}{\left|r-r_{0}\right|^{2}}+\frac{\mu_{2}}{\left|r+r_{0}\right|^{2}}\right)^{2}+\frac{16 \mu_{1} \mu_{2} X^{2} r_{2}^{2}}{\left|r-r_{0}\right|^{4}\left|r+r_{0}\right|^{4}}$.

The gravitational lensing time delay between two images $r_{a}, r_{b}$ can be calculated from (Schneider \& Weiss 1986)

$c \Delta t=\beta_{\mathrm{E}}^{2} \frac{D_{\mathrm{s}}}{D_{\mathrm{d}} D_{\mathrm{ds}}}\left(1+z_{\mathrm{d}}\right)\left[\Theta\left(r_{a}, x\right)-\Theta\left(r_{b}, x\right)\right]$,

where $\Psi(r)$ is the gravitational potential of the mass distribution and $\Theta(r, x)=(r-x)^{2} / 2-\Psi(r), D_{\mathrm{d}}$ is the angular diameter distance from the observer to the lens, $D_{\mathrm{s}}$ is the angular diameter distance from the observer to the source, and $D_{\mathrm{ds}}$ is angular diameter distance from the lens to the source.

In order to find all of the image positions for any given source position, we follow the search routine for image positions proposed in Schneider \& Weiss (1986). Firstly, we choose special starting point $x_{0}$ which has image positions $r_{0}$ solved using an analytical method. These starting points satisfy $x_{01}=0$ or $x_{02}=0$ where $x_{0}=\left(x_{01}, x_{02}\right)$. Secondly, let $x_{1}$ be a point near $x_{0}$. The corresponding image positions of $r_{1}$ are given by the differential lens equation, $\mathrm{d} x=A \mathrm{~d} r$, that is, $r_{1}^{\prime}=r_{0}+A^{-1}\left(r_{0}\right)\left(x_{1}-x_{0}\right)$. Thirdly, we insert $r_{1}^{\prime}$ into the lens Eq. (3) to find the corresponding source position $x_{1}^{\prime}=x\left(r_{1}^{\prime}\right)$. The condition is $\left|x_{1}-x_{1}^{\prime}\right|<\varepsilon$. If this condition is not satisfied, we take the next iterative step. For example, we use $r_{1}^{\prime}, x_{1}^{\prime}$ as starting points to do the next loop of calculation. Another thing to note is that any steps of the calculation should not cross a critical line, which is given by $\operatorname{det} A=0$. In order to find all image positions of a given source, we should choose suitable starting points in different areas separated by critical lines.

With a specific configuration of lenses and source, we require two conditions. Firstly, we should observe at least three images, that is, three peaks in the FRB light curve. The flux ratio $I$ between every two images should be smaller than a critical flux ratio $I_{f}$. Secondly, there are at least three images which have the observed time delay between every two images larger than a critical time $t_{f}$. Given the position of source and the distance of source and lenses, these conditions require the lens system to stay in some specific areas. We define these specific areas as the cross section $\sigma$. We use the Monte Carlo method to calculate the cross section. 
Table 1. Integrated optical depth $\bar{\tau}$ for different parameters for a FRB number density proportional to SFH.

\begin{tabular}{ccccccc}
\hline \hline$t_{f}, I_{f}$ & $10^{-4} \mathrm{~s}, 10^{3}$ & $10^{-3} \mathrm{~s}, 10^{3}$ & $10^{-4} \mathrm{~s}, 10^{2}$ & $10^{-3} \mathrm{~s}, 10^{2}$ & $10^{-4} \mathrm{~s}, 10^{1}$ & $10^{-3} \mathrm{~s}, 10^{1}$ \\
\hline$f=10^{-3}$ & $1.57 \times 10^{-4}$ & $9.50 \times 10^{-5}$ & $2.39 \times 10^{-5}$ & $1.05 \times 10^{-5}$ & $1.24 \times 10^{-6}$ & $4.81 \times 10^{-7}$ \\
$f=10^{-2}$ & $5.53 \times 10^{-3}$ & $3.19 \times 10^{-3}$ & $8.15 \times 10^{-4}$ & $3.42 \times 10^{-4}$ & $4.36 \times 10^{-5}$ & $1.67 \times 10^{-5}$ \\
$f=10^{-1}$ & $5.74 \times 10^{-2}$ & $3.31 \times 10^{-2}$ & $8.44 \times 10^{-3}$ & $3.58 \times 10^{-3}$ & $4.62 \times 10^{-4}$ & $1.72 \times 10^{-4}$ \\
\hline
\end{tabular}

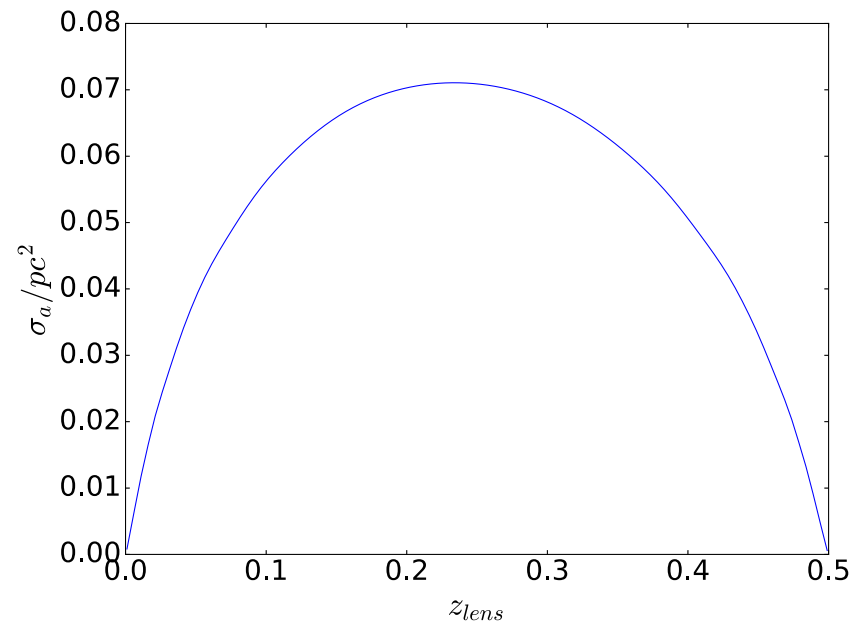

Fig. 1. Average cross section $\sigma_{a}$ of lenses at different distances when the redshift of the source is $z=0.5$ and the fraction of dark matter in PBHs is $f=0.1$. The critical time $t_{f}=0.1 \mathrm{~ms}$ and critical flux ratio $I_{f}=1000$ are used.

The separation of two lenses could affect the cross section. We generate the $\mathrm{PBH}$ binaries and obtain the distribution of their separations using the mechanism proposed by Nakamura et al. (1997) and Sasaki et al. (2016). Then, given the distance of source and lenses, we calculate the average cross section by simply averaging the cross section of a large number of $\mathrm{PBH}$ binaries staying in this distance. Here, the average cross section $\sigma_{a}\left(M, \mu_{1}, \mu_{2}, z_{\mathrm{s}}, z_{1}\right)$ is a function of the total mass of the lenses $M$, mass ratios $\mu_{1}$ and $\mu_{2}$, redshift of source $z_{\mathrm{s}}$ and lens system $z_{1}$. We calculate the average cross section of lenses at different distances when the source is located at $z=0.5$ as an example. The result is shown in Fig. 1.

Next we calculate the lensing optical depth of a source, which indicates the probability that this source is lensed and the multipeak structure is observable, at redshift $z_{\mathrm{s}}$ from

$\tau=\int_{0}^{z_{\mathrm{s}}} \mathrm{d} X(z)(1+z)^{2} n_{\mathrm{L}} \sigma_{a}(z)$

To obtain the estimated number of detected lensing events of FRBs by PBH binaries, we need to convolve the optical depth for a single FRB with the redshift distribution of FRBs. Similar to Muñoz et al. (2016) and Caleb et al. (2016), we discuss two redshift distributions of FRBs. The first one is a constant comoving number density, that is, $\rho(z)=$ constant. Observations show that the host galaxy of FRB 121102 is a dwarf galaxy with low metallicity and prominent emission lines (Tendulkar et al. 2016), sharing similar properties with long gamma-ray bursts (GRBs). Meanwhile, long GRBs trace the star formation history (SFH; Wijers et al. 1998; Wanderman \& Piran 2010; Wang et al. 2015). If FRB 121102 is typical of the wider FRB population, the rate of FRB would trace the SFH. So we also consider that FRB number density is proportional to the SFH. The SFH is
(Hopkins \& Beacom 2006)

$\rho(z)=h \frac{a+b z}{1+\left(\frac{z}{c}\right)^{d}}$,

with $a=0.0170, b=0.13, c=3.3$, and $d=5.3$ and $h=0.7$ (Cole et al. 2001; Wang 2013) is used. The comoving volume of a shell of width $\mathrm{d} z$ at redshift $z$ is $\mathrm{d} V(z)=\left[4 \pi \chi^{2}(z) / H(z)\right] \mathrm{d} z$. Introducing the Gaussian cutoff to represent an instrumental signal-to-noise $(\mathrm{S} / \mathrm{N})$ threshold, we get the distribution of FRBs;

$N_{\text {FRB }}(z)=\mathbb{N}_{\text {FRB }} \frac{\rho(z) \chi(z)^{2}}{H(z)(1+z)} \mathrm{e}^{-d_{\mathrm{L}}^{2} /\left[2 d_{\mathrm{L}}^{2}\left(z_{\text {cut }}\right)\right]}$,

where $\mathbb{N}_{\text {FRB }}$ is the normalizing factor and the term $(1+z)$ accounts for the effect of cosmological time dilation. We choose $\Omega_{\mathrm{vac}}=0.714, \Omega_{\mathrm{M}}=0.286$, and $H_{0}=69.6 \mathrm{~km} \mathrm{~s}^{-1} \mathrm{Mpc}^{-1}$ in our calculation.

Then we can calculate the integrated optical depth $\bar{\tau}$

$\bar{\tau}=\int \mathrm{d} z \tau(z) N_{\mathrm{FRB}}(z)$

Inter-channel dispersion will broaden the FRB pulse width. Meanwhile, its intrinsic pulse profile and scattering with the intergalactic medium will also contribute the total pulse width. Similar to Muñoz et al. (2016), we choose 1 and $0.1 \mathrm{~ms}$ as the critical times $t_{f}$. For the critical flux ratio $I_{f}$, we adopt three different values, that is, $10^{3}, 100$, and 10 . The cutoff redshift is chosen as $z_{\text {cut }}=0.5$ (Muñoz et al. 2016), which fits the current FRB catalog well for both two distributions of FRBs. In Table 1, we show the integrated optical depth $\bar{\tau}$ for different fractions of dark matter $f=10^{-1}, 10^{-2}, 10^{-3}$ in PBHs for FRB number density proportional to SFH. In Table 2 , we show $\bar{\tau}$ for different $f=10^{-1}, 10^{-2}, 10^{-3}$ in PBHs for a constant FRB number density.

We choose the above values for $t_{f}$ and $I_{f}$ because the duration time of FRBs is on the order of $1 \mathrm{~ms}^{1}$. Therefore, the choice of critical time $t_{f}=1 \mathrm{~ms}$ is reasonable. We compare the repeating FRBs with the lensing FRBs since repeating FRBs also have multi-peak structures which might mix with the lensing FRBs. It turns out that the time interval between successive bursts for the repeating FRB 121102 is larger than several tens of seconds (Wang \& Yu 2017). Therefore, the lensing FRBs can be discriminated from typical repeating FRBs. To date, multiple bursts have only been observed for the repeating FRB 121102. Fluxes are $670 \mathrm{mJy}$ (Chatterjee et al. 2017) and $20 \mathrm{mJy}$ (Spitler et al. 2016) for the brightest and dimmest burst, respectively, giving a flux ratio between them of about 33. Therefore, the choice of the critical flux ratio $I_{f}$ is reasonable.

\footnotetext{
1 http://www.frbcat.org
} 
Table 2. Integrated optical depth $\bar{\tau}$ for different parameters for a constant FRB comoving number density.

\begin{tabular}{ccccccc}
\hline \hline$t_{f}, I_{f}$ & $10^{-4} \mathrm{~s}, 10^{3}$ & $10^{-3} \mathrm{~s}, 10^{3}$ & $10^{-4} \mathrm{~s}, 10^{2}$ & $10^{-3} \mathrm{~s}, 10^{2}$ & $10^{-4} \mathrm{~s}, 10^{1}$ & $10^{-3} \mathrm{~s}, 10^{1}$ \\
\hline$f=10^{-3}$ & $1.30 \times 10^{-4}$ & $7.92 \times 10^{-5}$ & $1.98 \times 10^{-5}$ & $8.85 \times 10^{-6}$ & $1.02 \times 10^{-6}$ & $4.03 \times 10^{-7}$ \\
$f=10^{-2}$ & $4.59 \times 10^{-3}$ & $2.67 \times 10^{-3}$ & $6.79 \times 10^{-4}$ & $2.89 \times 10^{-4}$ & $3.59 \times 10^{-5}$ & $1.41 \times 10^{-5}$ \\
$f=10^{-1}$ & $4.76 \times 10^{-2}$ & $2.77 \times 10^{-2}$ & $7.02 \times 10^{-3}$ & $3.02 \times 10^{-3}$ & $3.80 \times 10^{-4}$ & $1.46 \times 10^{-4}$ \\
\hline
\end{tabular}

Table 3. Results of the simulation of $2 \times 10^{5} \mathrm{FRBs}$ for different parameters.

\begin{tabular}{ccccccc}
\hline \hline$t_{f}, I_{f}$ & $10^{-3} \mathrm{~s}, 10^{1}$ & $10^{-4} \mathrm{~s}, 10^{1}$ & $10^{-3} \mathrm{~s}, 10^{2}$ & $10^{-4} \mathrm{~s}, 10^{2}$ & $10^{-3} \mathrm{~s}, 10^{3}$ & $10^{-4} \mathrm{~s}, 10^{3}$ \\
\hline \multicolumn{7}{c}{ Choosing the smallest $b / \Sigma_{\mathrm{cr}}^{-1}$} \\
$f=10^{-3}$ & 0 & 0 & 0 & 0 & 3 & 7 \\
$f=10^{-2}$ & 0 & 1 & 7 & 34 & 121 & 257 \\
$f=10^{-1}$ & 0 & 9 & 82 & 275 & 1085 & 2256 \\
\hline \multicolumn{7}{c}{ Choosing the smallest $b / \sqrt{\Sigma_{\mathrm{cr}}^{-1}}$} \\
$f=10^{-3}$ & 0 & 0 & 3 & 5 & 5 & 8 \\
$f=10^{-2}$ & 0 & 2 & 16 & 38 & 125 & 257 \\
$f=10^{-1}$ & 0 & 6 & 98 & 296 & 1079 & 2281 \\
\hline & 0 & \multicolumn{7}{c}{} \\
$f=10^{-3}$ & 0 & 0 & 0 & 0 & \\
$f=10^{-2}$ & 0 & 0 & 14 & 34 & 115 & 241 \\
$f=10^{-1}$ & 0 & 8 & 108 & 284 & 1023 & 2129 \\
\hline
\end{tabular}

Since the integrated optical depth $\bar{\tau}$ is very small, the expected number of lensing FRBs by $\mathrm{PBH}$ binaries is

$N_{\text {exp }}=N \times P=N \times\left(1-\mathrm{e}^{-\tau}\right)=N \times \tau$.

Connor et al. (2016) estimated that the Canadian hydrogen intensity mapping experiment (CHIME) could detect about $10^{4}$ FRBs per year. We could expect the detection of FRBs strongly lensed by $\mathrm{PBH}$ binaries. For example, the number of strongly lensed FRBs is about 36 for $t_{f}=1 \mathrm{~ms}, I_{f}=100$ and $f=0.1$ per year. Because most of the FRBs are below $z=1.5$ in our calculation when choosing $z_{\text {cut }}=0.5$, the band of CHIME is not severely affected by redshift.

\section{Numerical simulations}

We further simulate the gravitational lensing events of FRBs by PBH binaries to verify the analytical calculation in the above section. Assuming the distribution of $\mathrm{PBH}$ binaries is isotropic and uniform in space, we generate the PBH binaries following Sasaki et al. (2016). As mentioned in the previous section, we consider the distribution of FRBs following the SFH (Caleb et al. 2016). For the constant comoving number density, the difference of the simulation resulting from the SFH case is less than $30 \%$.

We simulate $2 \times 10^{5}$ FRBs for different fractions of dark matter $f=10^{-1}, 10^{-2}, 10^{-3}$ in PBHs, respectively. We choose $\Omega_{\mathrm{vac}}=0.714, \Omega_{\mathrm{M}}=0.286$, and $H_{0}=69.6 \mathrm{~km} \mathrm{~s}^{-1} \mathrm{Mpc}^{-1}$ in our calculation. For each FRB, we consider the PBH binaries which could provide the strongest lensing effect. The lensing efficiency is indicated by lensing kernel

$\Sigma_{\mathrm{cr}}^{-1}=\frac{4 \pi G D_{\mathrm{ds}} D_{\mathrm{d}}}{D_{\mathrm{s}}}$,

which is proportional to $D_{\mathrm{ds}} D_{\mathrm{d}} / D_{\mathrm{s}}$ (Sheldon 2002; Courbin \& Minniti 2002). It describes the geometry of the lense-source system. A larger $\Sigma_{\mathrm{cr}}^{-1}$ indicates a stronger lensing effect. As an example, the lensing kernel of the lenses at different redshifts when the source is at redshift $z=1.3$ is shown in Fig. 2. The distance of PBH binaries from the line of sight can also affect the lensing effect. Because the lensing events depend on other parameters, such as the orbital elements of the binaries, the strongest lensing event might not give the expected observable multi-peak FRBs. Meawhile, the magnification and time delay are also required. Therefore, we use several criteria to show the reliability of our results. We choose the $\mathrm{PBH}$ binaries by finding the smallest value of $\Delta \theta / \sqrt{\Sigma_{\mathrm{cr}}^{-1}}, b / \sqrt{\Sigma_{\mathrm{cr}}^{-1}}$ or $b / \Sigma_{\mathrm{cr}}^{-1}$, where $\theta$ is the angular distance of the PBH binary from the line of sight, and $b$ is the linear distance of the PBH binary from the line of sight, that is, the impact parameter. We calculate the positions of images, time delay, and flux ratio of every second image. Then, we check whether they could conform with the conditions concerning time delay and flux ratio. We record all the lensing events with flux ratio $I<10^{4}$ and time delay $t>0.5 \times 10^{-4} \mathrm{~s}$ and give the results of the simulations in Table 3 for all three different ways of choosing PBH binaries. We find that the difference due to this latter choice is not obvious. Therefore, the choice of criteria cannot significantly affect the results. They all provide a lower limit of observable multi-peak FRBs. We further show the results for choosing $\mathrm{PBH}$ binaries by finding the smallest value of $b / \Sigma_{\mathrm{cr}}^{-1}$ in Fig. 3. The number of lensing events is strongly influenced by the fraction of dark matter, $f$, critical time delay, $t_{f}$, and critical flux ratio, $I_{f}$.

There are some differences between the analytical results in Sect. 2 and numerical simulations in Sect. 3. These differences are reasonable because we make some assumptions in the calculation in Sect. 2. For example, the average cross section used to calculate the optical depth is not very accurate. In Sect. 3, we use the Monte Carlo method to calculate the cross section since the allowed regions are not regular, as only a single lense is used. 


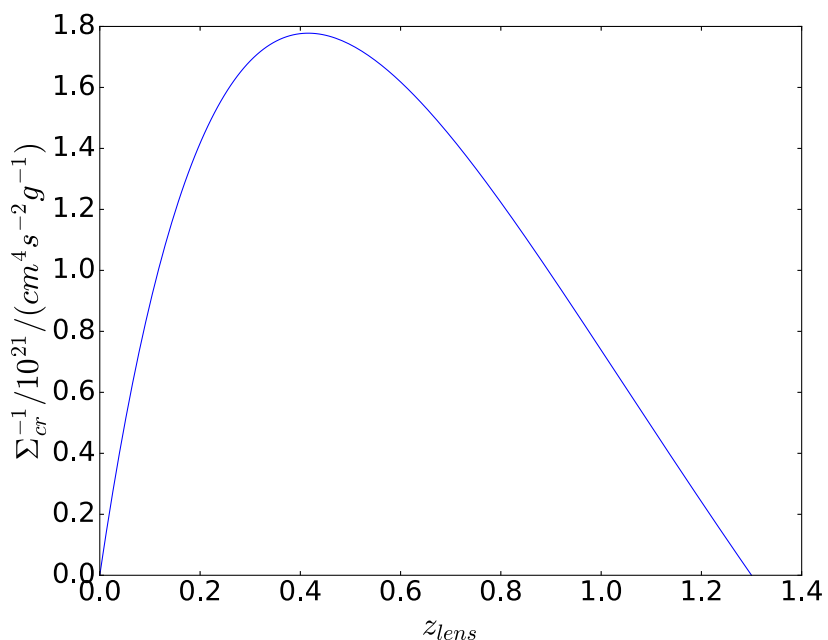

Fig. 2. Lensing kernel $\Sigma_{\text {cr }}^{-1}$ of lenses at different redshifts when the redshift of the source is $z=1.3$.

\section{Discussion and conclusion}

FRBs are promising tools to probe MACHOs. Firstly, FRBs are strong and short events, which means that they could serve as good candidates to probe the lensing events. As a result of lensing by black hole binaries, we should detect multi-peak FRBs. Since FRBs are strong and short, their multi-peak structure might be observable. Secondly, the rate of FRBs can be as high as a few thousand per day for the whole sky. Lastly, in the upcoming or ongoing surveys, the observation of a large number of FRBs is promising.

In this paper, we estimate the possibility of detecting lensing events of FRBs by PBH binaries. The typical time interval between different images is roughly $1 \mathrm{~ms}$. The flux ratio between different images is from approximately 10 to $10^{3}$. With an expected detection rate of FRBs by the upcoming experiment CHIME of $10^{4}$ FRBs per year, we could expect five detections of multi-peak FRBs with time intervals larger than $1 \mathrm{~ms}$ and flux ratios less than $10^{3}$ per year in the upcoming FRB surveys if the fraction of dark matter in MACHOs is $f=0.01$. If the lensing events are detected, we could analyze the structure of the peaks of FRBs to infer the separation and masses of two lenses to verify the primordial black hole scenario for gravitational-wave events. Alternatively, if no FRBs are lensed, the strongest constraints will be those put on the fraction of dark matter in the form of compact objects.

In our calculation, we use the formation mechanism of $\mathrm{PBH}$ binaries proposed in Nakamura et al. (1997). Tidal force from the nearby $\mathrm{PBH}$ provides the angular momentum of the $\mathrm{PBH}$ binaries. Bird et al. (2016) proposed that PBH binaries are formed due to the gravitational radiation when they pass by one another. We wish to point out that in our calculation, these two mechanisms do not have obvious differences. In the first mechanism, most of the $\mathrm{PBH}$ binaries will have an eccentricity very close to one. The movement of PBHs in the binary system could be very similar to movement when they are free. If we assume the same number density of PBHs, the results from two mechanisms would be very similar.

Cordes et al. (2017) claim that plasma lenses in FRB host galaxies can produce multiple bursts with different apparent strengths, arrival times, and dispersion measurements. There are several differences between our results and theirs. Firstly, the positions and "gain" (or amplification) of burst images are
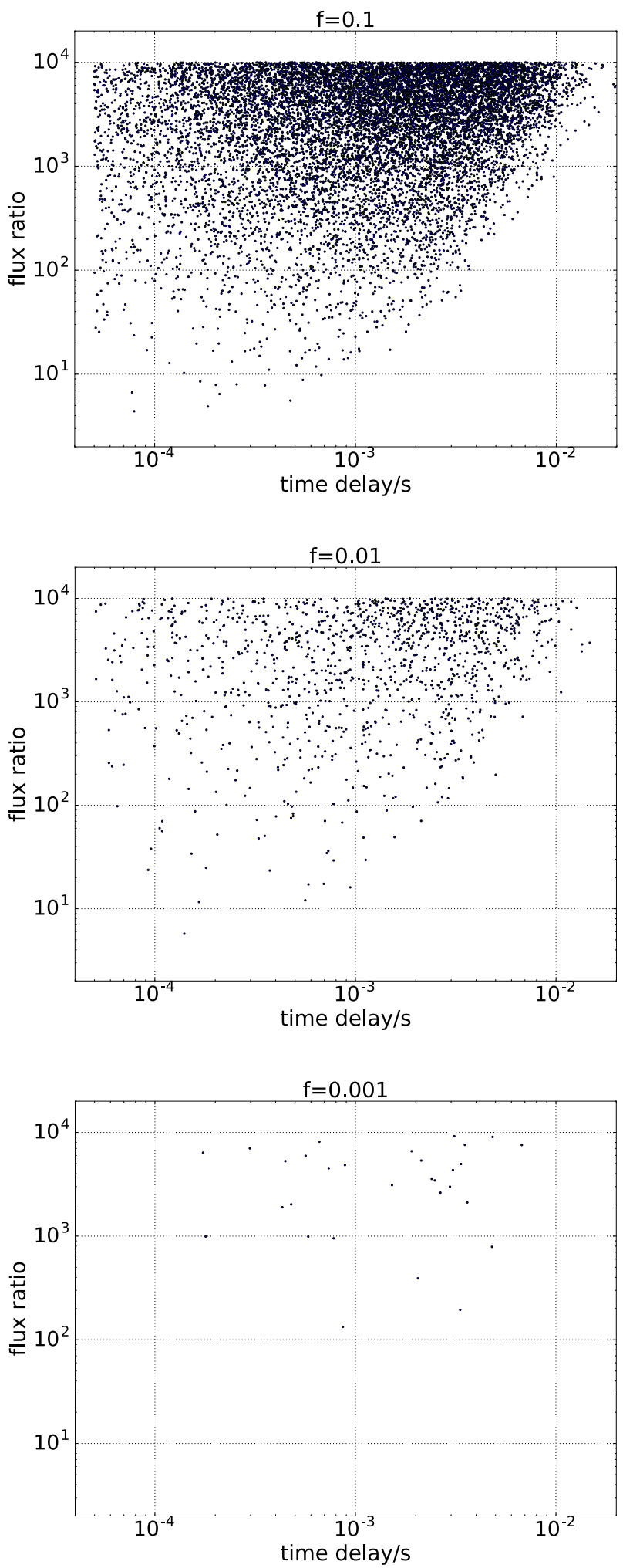

Fig. 3. Simulations of $2 \times 10^{5}$ FRBs with different fractions of dark matter $f=10^{-1}, 10^{-2}, 10^{-3}$, respectively. All three panels show all the lensing events with flux ratio $I<10^{4}$ and time delay $t>0.5 \times 10^{-4} \mathrm{~s}$.

different for different frequencies in Cordes et al. (2017). However, in our discussion, the lensing effect caused by $\mathrm{PBH}$ binaries would be the same for any frequency. Secondly, the DMs of different images of bursts are different in their calculations. As for images caused by PBH lensing, the DMs of burst images contributed by the host galaxy are the same. Thirdly, their theory 
allows for the existence of larger time intervals between images, which could interpret the repeating FRB 121102 qualitatively. In our paper, we expect to distinguish between repeating FRBs and multiply imaged bursts caused by PBH binaries.

Acknowledgements. We thank the anonymous referee for constructive suggestions that have allowed us to improve our manuscript. This work is supported by the National Basic Research Program of China (973 Program, grant No. 2014CB845800) and the National Natural Science Foundation of China (grants 11422325 and 11373022), the Excellent Youth Foundation of Jiangsu Province (BK20140016).

\section{References}

Abbott, B. P., Abbott, R., Abbott, T. D., et al. 2016a, Phys. Rev. Lett., 116, 061102 Abbott, B. P., Abbott, R., Abbott, T. D., et al. 2016b, Phys. Rev. Lett., 116, 241103 Alcock, C., Allsman, R. A., Alves, D. R., et al. 2001, ApJ, 550, 169 Ali-Haïmoud, Y., \& Kamionkowski, M. 2017, Phys. Rev. D, 95, 043534 Arvanitaki, A., Baryakhtar, M., Dimopoulos, S., Dubovsky, S., \& Lasenby, R. 2017, Phys. Rev. D, 95, 043001

Bandura, K., Addison, G. E., Amiri, M., et al. 2014, Proc. SPIE, 9145, 914522

Belczynski, K., Holz, D. E., Bulik, T., \& O’haughnessy, R. 2016, Nature, 534, 512

Belotsky, K. M., Dmitriev, A. E., Esipova, E. A., et al. 2014, Mod. Phys. Lett. A, 29, 1440005

Bird, S., Cholis, I., Muñoz, J. B., et al. 2016, Phys. Rev. Lett., 116, 201301

Caleb, M., Flynn, C., Bailes, M., et al. 2016, MNRAS, 458, 718

Carr, B. J. 1975, ApJ, 201, 1

Carr, B. J. 1976, ApJ, 206, 8

Carr, B. J., \& Hawking, S. W. 1974, MNRAS, 168, 399

Chatterjee, S., Rodriguez, C. L., \& Rasio, F. A. 2017, ApJ, 834, 68

Cholis, I., Kovetz, E. D., Ali-Haïmoud, Y., et al. 2016, Phys. Rev. D, 94, 084013

Cole, S., Norberg, P., Baugh, C. M., et al. 2001, MNRAS, 326, 255

Connor, L., Lin, H. H., \& Masui, K. 2016, MNRAS, 460, 1054

Cordes, J. M., Wasserman, I., Hessels, J. W. T., et al. 2017, ApJ, 842, 35

Courbin, F., \& Minniti, D. 2002, Gravitational Lensing: An Astrophysical Tool (Berlin: Springer Science \& Business Media)

Dai, Z. G., Wang, J. S., Wu, X. F., \& Huang, Y. F. 2016, ApJ, 829, 27

Falcke, H., \& Rezzolla, L. 2014, A\&A, 562, A137

Frampton, P. H., Kawasaki, M., Takahashi, F., \& Yanagida, T. T. 2010, JCAP, 04, 023

García-Bellido, J., Linde, A., \& Wands, D. 1996, Phys. Rev. D, 54, 6040

Hartwig, T., Volonteri, M., Bromm, V., et al. 2016, MNRAS, 460, L74

Hopkins, A. M., \& Beacom, J. F. 2006, ApJ, 651, 142
Hosokawa, T., Hirano, S., Kuiper, R., et al. 2016, ApJ, 824, 119

Inayoshi, K., Kashiyama, K., Visbal, E., \& Haiman, Z. 2016, MNRAS, 461, 2722 Khlopov, M. Y. 2010, Res. Astron. Astrophys., 10, 495

Lorimer, D. R., Bailes, M., McLaughlin, M. A., Narkevic, D. J., \& Crawford, F. 2007, Science, 318, 777

Mandic, V., Bird, S., \& Cholis, I. 2016, Phys. Rev. Lett., 117, 201102

Mediavilla, E., Muñoz, J. A., \& Falco, E., et al. 2009, ApJ, 706, 1451

Mészáros, P. 1975, A\&A, 38, 5

Mink, S. E., \& Mandel, I. 2016, MNRAS, 460, 3545

Monroy-Rodríguez, M. A., \& Allen, C. 2014, ApJ, 790, 159

Muñoz, J. B., Kovetz, E. D., Dai, L., \& Kamionkowski, M. 2016, Phys. Rev. Lett., 117,091301

Nakamura, T., Sasaki, M., Tanaka, T., \& Thorne, K. S. 1997, ApJ, 487, 139

Newburgh, L. B., Bandura, K., Bucher, M. A., et al. 2016, SPIE Conf. Ser., 9906, 99065X

Pooley, D., Rappaport, S., Blackburne, J., et al. 2009, ApJ, 697, 1892

Quinn, D. P., Wilkinson, M. I., Irwin, M. J., et al. 2009, MNRAS, 396, 11

Raccanelli, A., Kovetz, E. D., Bird, S., et al. 2016, Phys. Rev. D, 94, 023516

Ricotti, M. 2007, ApJ, 662, 53

Ricotti, M., Ostriker, J. P., \& Mack, K. J. 2008, ApJ, 680, 829

Rodriguez, C. L., Chatterjee, S., \& Rasio, F. A. 2016a, Phys. Rev. D, 93, 084029

Rodriguez, C. L., Haster, C. J., Chatterjee, S., et al. 2016b, ApJ, 824, L8

Sasaki, M., Suyama, T., Tanaka, T., et al. 2016, Phys. Rev. Lett., 117, 061101

Schneider, P., \& Weiß, A. 1986, A\&A, 164, 237

Schutz, K., \& Liu, A. 2017, Phys. Rev. D, 95, 023002

Sheldon, E. S. 2002, PhD Thesis, University of Michigan, USA

Spitler, L. G., Scholz, P., Hessels, J. W. T., et al. 2016, Nature, 521, 202

Tendulkar, S. P., Bassa, C. G., Chatterjee, S., et al. 2017, ApJ, 834, L7

Thornton, D., Stappers, B., Bailes, M., et al. 2013, Science, 341, 53

Totani, T. 2013, PASJ, 65, L12

Tisserand, P., Guillou, L. L., Afonso, C., et al. 2007, A\&A, 469, 387

Verheijen, M. A. W., Oosterloo, T. A., van Cappellen, W. A., et al. 2008, AIP Conf. Proc., 1035, 265

Wanderman, D., \& Piran, T. 2010, MNRAS, 406, 1944

Wang, F. Y. 2013, A\&A, 556, A90

Wang, F. Y., \& Yu, H. 2017, JCAP, 03, 023

Wang, F. Y., Dai, Z. G., \& Liang, E. W. 2015, New Astron. Rev., 67, 1

Wang, J. S., Yang, Y. P., Wu, X. F., Dai, Z. G., \& Wang, F. Y. 2016, ApJ, 822, L7

Wijers, R. A. M. J., Bloom, J. S., Bagla, J. S., et al. 1998, MNRAS, 294, L13 Woosley, S. E. 2016, ApJ, 824, L10

Wyrzykowski, L., Skowron, J., Kozlowski, S., et al. 2011, MNRAS, 416, 2949

Yoo, J., Chaname, J., \& Gould, A. 2004, ApJ, 601, 311

Yu, H., \& Wang, F. Y. 2017, A\&A, 660, A3

Yu, Y.-W., Cheng, K.-S., Shiu, G., \& Tye, H. 2014, JCAP, 11, 040

Zhang, B. 2014, ApJ, 780, L21

Zhang, B. 2016, ApJ, 827, L31 